\title{
Initial stage of plate lifting from a water surface
}

\author{
Alexander Korobkin • Tatyana Khabakhpasheva • \\ Javier Rodríguez-Rodríguez
}

Received: 20 January 2015 / Accepted: 6 November 2015 / Published online: 26 December 2015

(C) The Author(s) 2015. This article is published with open access at Springerlink.com

\begin{abstract}
This study deals with the flow induced by a rigid flat plate of finite length, initially touching a horizontal water surface, when it starts to move upwards with constant acceleration. In the present model, negative hydrodynamic pressures on the lower (wetted) surface of the plate are allowed, and thus, the water follows the plate due to the resulting suction force. The acceleration of the plate and the plate length are such that gravity, surface tension and viscous effects can be neglected during the early stages of the motion. Under these assumptions, the initial two-dimensional, potential flow caused by the plate lifting is obtained by using the small-time expansion of the velocity potential. This small-time solution is not valid close to the plate edges, as it predicts there singular flow velocities and unbounded displacements of the water-free surface. It is shown that close to the plate edges the flow is nonlinear and self-similar to leading order. This nonlinear flow is computed by the boundary-element method combined with a time-marching scheme. The numerical time-dependent solution approaches the self-similar local solution with time.
\end{abstract}

Keywords Free-surface flows $\cdot$ Matched asymptotics $\cdot$ Numerical methods $\cdot$ Water exit

Mathematics Subject Classification $76 \mathrm{~B} 10$

\section{Introduction}

The unsteady two-dimensional flow caused by the impulsive vertical motion of a floating rigid plate is considered. Initially, the liquid occupies the lower half-plane and is at rest. The draft of the floating plate is much smaller that the plate length, $2 L$, and is neglected in the present analysis. The body starts to move suddenly upwards from the liquid at a constant acceleration $a$, which is assumed to be much greater than the gravitational acceleration $g$. The liquid is assumed to be ideal and incompressible with negligible surface tension. Consequently, the generated flow can be modelled as symmetric and potential. We shall determine the flow during the early stages of the motion.

A. Korobkin $(\varangle) \cdot$ T. Khabakhpasheva

School of Mathematics, University of East Anglia, Norwich, UK

e-mail: a.korobkin@uea.ac.uk

J. Rodríguez-Rodríguez

Fluid Mechanics Group, Universidad Carlos III de Madrid, Madrid, Spain 
The present study is motivated by the experiments performed by Reis et al. [1] with a glass disc representing the tip of a cat's tongue. The disc was initially placed on the still water surface, with a negligible draft, and then pulled vertically upwards. The motion of the disc reproduced the motion of the cat's tongue recorded with a high-speed camera, which followed the time-dependent acceleration. In [1], the authors claimed that "the fluid dynamics of lapping is governed by inertia and gravity, whereas viscous and capillary forces are negligible".

A linearized model of water exit was developed in [2] by means of the results and ideas from [1]. In this model, the hydrodynamic equations and boundary conditions were linearized, which is acceptable during the initial stage of the flow, and the unknown size of the wetted surface was suggested to be determined by using the condition that the speed of the wetted area shrinking is proportional to the local velocity of the flow at its periphery. Comparing the analytical results provided by the model from [2] with the numerical results by Piro and Maki [3], it was found that the coefficient of proportionality is equal to two. This value of the coefficient was used in $[4,5]$ to calculate the hydrodynamic forces acting on two-dimensional and axisymmetric bodies exiting from a water surface and to compare the theoretical results with the numerical ones computed by fully nonlinear numerical simulations. Note that this value of the coefficient has not been justified. More research is needed to explain this value and conditions of exit leading to this value. The theoretical model from [2] does not account for the shape of the body, gravity, surface tension or liquid viscosity. Nevertheless, its predictions compare well with CFD results, using the same value of the coefficient of proportionality for all the cases.

The theoretical model requires the solution of a nonlinear and singular integral equation with respect to the time-dependent size of the wetted area. It was discovered in [2] that both the solution and the convergence of the algorithm used for its computation are strongly dependent on an initial condition that must be obtained by examining the initial stages of the flow. Specifically, the integral equation (2.25) from [2] could not be solved numerically on its own without the asymptotic formula (3.11). The initial motion of the lifting body is also important for lapping, which is possible only for specific lifting-velocity laws. If the lifting is too slow, gravity could preclude the liquid to be lifted up to a required height. In contrast, if the body motion is too fast, cavitation may occur at the wetted surface, and the body may separate from the liquid at early stages. A successful law of a disc motion, when the liquid is lifted by the disc up to a significant height, can be found in [1].

As a consequence of its relevance to the naval and aeronautical industry, as well as to understand some fundamental questions in natural sciences (such as the cat lapping problem), the water-exit problem has been explored extensively in the past. Greenhow [6] and Greenhow and Moyo [7] studied the exit of initially submerged bodies by numerical methods. Oliver [8] was concerned with the stability of the advancing liquid-solid contact line. Baarholm [9], Scolan et al. [10] and Faltinsen et al. [11] considered the problem of wave impact from below on a platform placed just above the water surface. Piro and Maki $[3,12]$ analysed the combined entry-exit problem, using computational fluid mechanics with application to high-speed boats. Indeed, some of the analyses performed for the entry problem can be applied to the exit counterpart [13]. Finally, Tassin et al. [14] were concerned with the hydrodynamic loads in the rear part of the fuselage during aircraft ditching on water. See also the references in $[3,12,14]$ about the relation of the exit problem to the naval and aeronautical fields.

The configuration of the problem is such that the plate motion upwards does not generate a flow if there is no adhesion force between the fluid and the surface of the plate. This configuration is different from those studied in $[15,16]$, where an initially submerged circular cylinder exits from the liquid piercing its free surface. We consider a plate motion with large acceleration such that the gravity acceleration can be neglected. For slow motions of the plate, gravity plays an important role and the present model is not applicable. The results of the present paper show that the hydrodynamic pressure is below the atmospheric pressure at any point of the contact region. This explains the shrinking of the contact region in time. This phenomenon is known as ventilation. However, separation of the liquid from the lower surface of the plate does not occur instantly. The speed of the contact region shrinking depends on the physical characteristics of the liquid, the plate surface and the plate motion. These physical properties are important close to the plate surface but can be neglected far from the surface, as was argued in [1].

The analysis of the present paper is restricted to the initial stage of the flow, when the plate displacement is small compared with the plate length. Asymptotic methods are used to obtain a uniformly valid initial asymptotic solution. The approach is similar to that developed by Iafrati and Korobkin $[17,18]$ for the problem of initial flow caused 
by sudden motion of a floating body. In this approach, the flow region is divided into the main flow region, where the solution can be obtained by the small-time expansion method, and the immediate vicinities of the plate edges, where the inner leading-order solution is obtained numerically. This inner solution is then matched to the small-time solution in the main region. The matching procedure provides the far-field condition for the inner solution.

The problem is formulated in Sect. 2 with respect to the velocity potential and the shape of the liquid free surface in non-dimensional variables. Conditions on the parameters of the problem, which allow us to neglect viscosity of the liquid, surface tension on the liquid free surface and the gravity are derived at the end of Sect. 2. The secondorder small-time solution of the problem is obtained in Sect. 3 together with the corresponding eigen solution. The equations and boundary conditions governing the flow close to the plate edge during the initial stage are derived in Sect. 4. Theoretical analysis of the inner solution is provided. The numerical solution of the inner-boundary problem is presented in Sect. 5. Finally, the conclusions are drawn and future work is discussed in Sect. 6.

\section{Formulation of the problem}

The two-dimensional unsteady problem of a plate lifting from the water surface is formulated in Cartesian coordinates and in non-dimensional variables. Initially, a plate of length $2 L$ touches the still and horizontal water surface. Then the plate starts to move suddenly upwards at a constant acceleration $a$. The half-width of the plate $L$ is taken as the length scale, and the time scale $T$ is such that the displacement of the plate, which is of order $a T^{2}$, is much smaller than the length scale of the problem, $a T^{2} \ll L$. The velocity scale is $a T$, and the pressure scale is $\rho a L$, where $\rho$ is the liquid density. The ratio $\varepsilon=a T^{2} / L$ plays the role of a small parameter of the problem. Gravity and surface tension are not included in the present model.

Initially, the liquid is at rest and occupies the lower half-plane, $y<0$. The interval $-1<x<1$ on the liquid boundary, where $y=0$, corresponds to the initial position of the plate. The midpoint of the plate is taken as the origin of the coordinate system $x y$. The position of the plate at time $t$ is given by $y=\frac{1}{2} \varepsilon t^{2},|x|<1$. Assuming that the wetted part of the plate is shrinking in time, we introduce a function $c(t)$ such that the interval $|x|<c(t, \varepsilon)$, $c(0, \varepsilon)=1$, corresponds to the wetted part of the plate. The flow is assumed two-dimensional, symmetric with respect to the vertical line $x=0$, and potential with velocity potential $\varphi(x, y, t)$. The scale of this velocity potential is $a T L$. The hydrodynamic pressure $p(x, y, t)$ is given by the Bernoulli equation

$p=-\varphi_{t}-\frac{1}{2} \varepsilon|\nabla \varphi|^{2}$,

in the flow region $\Omega(t, \varepsilon)$. The position of the liquid free surface is described by $y=\varepsilon \zeta(x, t),|x|>c(t, \varepsilon)$. The scale of the free-surface deflection is $a T^{2}$. The velocity potential satisfies the Laplace equation and the following boundary conditions:

$$
\begin{aligned}
& \nabla^{2} \varphi=0 \quad \text { in } \Omega(t, \varepsilon), \\
& \varphi_{y}=t \quad\left(y=\frac{1}{2} \varepsilon t^{2},|x|<c(t, \varepsilon)\right), \\
& \zeta_{t}+\varepsilon \zeta_{x} \varphi_{x}=\varphi_{y}, \quad \varphi_{t}+\frac{1}{2} \varepsilon|\nabla \varphi|^{2}=0 \quad(y=\varepsilon \zeta(x, t),|x|>c(t, \varepsilon)), \\
& \varphi(x, y, t) \rightarrow 0, \quad \zeta(x, t) \rightarrow 0 \quad\left(x^{2}+y^{2} \rightarrow \infty\right), \\
& \varphi(x, y, 0)=0, \quad(y<0), \quad \zeta(x, 0)=0 \quad(|x|>1) .
\end{aligned}
$$

Here (3) is the body boundary condition imposed on the moving plate; conditions (4) correspond to the kinematic and dynamic boundary conditions on the free surface; the far-field conditions (5) imply that the flow caused by the lifting plate decays at infinity; and the initial conditions (6) imply that initially the liquid is at rest and its upper boundary coincides with $y=0$. The small parameter $\varepsilon$ appears in these equations in such a way that the nonlinear terms can be neglected in the leading order as $\varepsilon \rightarrow 0$, and the boundary conditions can be imposed at the initial liquid level, $y=0$. 
In the formulation (2)-(6), there is still no equation which determines the motion of the contact points $x=$ $\pm c(t, \varepsilon)$. It is not clear at present which condition should be imposed at the contact point. Baarholm [9] in his numerical analysis of wave impacts used the condition that the speeds of the contact points are equal to the local velocities of the flow at these points; that is, the contact points are material points. In the linearized model of water exit [2], the speeds of the contact points were assumed to be proportional to the local velocities of the flow at these points. The coefficient of proportionality was chosen as 2 using the CFD results from [3]. In previous studies, it was noticed that the speeds of the contact points are small during the initial stage of exit, $c^{\prime}(t)=O\left(t^{1 / 3}\right)$ as $t \rightarrow 0$ (see [2]). This result allows us to neglect the motion of the contact points during the early stage of the plate lifting in the calculation of the flow far from the plate edges. However, close to the edges, the motions of the contact points should be taken into account as it will be shown later. We will investigate the effect of the motion of the contact point on the flow close to the plate edge.

In the present study, we are concerned with the asymptotic solution of the problem (2)-(6) accurate up to $o(\varepsilon)$ as $\varepsilon \rightarrow 0$ in the main flow region. The viscous term $v \nabla^{2} \vec{v}$ in the Navier-Stokes equation, where $v$ is the kinematic viscosity of the liquid, can be neglected compared with the inertia term $\vec{v}_{t}$ and the convective term $(\vec{v} \cdot \nabla) \vec{v}$ if $\nu T / L^{2} \ll \varepsilon$, which gives $T \gg v / a L$. For $v \approx 10^{-6} \mathrm{~m} \mathrm{~s}^{-1}$ (water at $20^{\circ} \mathrm{C}$ ), $L=5 \mathrm{~cm}$ and $a=20 \mathrm{~m} \mathrm{~s}^{-2}$, the inequality gives $T \gg 10^{-6} \mathrm{~s}$. In the immediate vicinities of the plate edges, the viscous effects are expected to be more important than in the main flow region. However, it can be shown that the liquid viscosity can be neglected at leading order, even in the immediate vicinities when $T \gg 10^{-5} \mathrm{~s}$ for the same conditions. Therefore, the viscous effects are important only during the very early stages of the flow, the duration of which can be estimated as $10^{-5} \mathrm{~s}$.

It can be shown that the surface tension can be neglected in the main part of the free surface with accuracy $o(\varepsilon)$ and in the immediate vicinities of the plate edges at leading order after a very short initial stage of duration $10^{-3} \mathrm{~s}$. Indeed, in the main part of the flow region, the pressure is of the order of $\rho a L$, whereas the capillary pressure is of order $\sigma / L$, where $\sigma$ is the surface tension coefficient. Thus, the ratio $\sigma / L \ll \varepsilon$ when $T^{2} \gg \sigma /\left(\rho a^{2} L\right)$. This inequality gives $T \gg 1.9 \times 10^{-3}$ s for water of density $\rho=1000 \mathrm{~kg} \mathrm{~m}^{-3}$ and surface tension coefficient $\sigma \approx 72 \times 10^{-3} \mathrm{~N} \mathrm{~m}^{-1}$. As will be shown here, the pressure is of order of $\rho a L \varepsilon^{1 / 3}$ in the vicinity of the plate edge, the size of which is of order $L \varepsilon^{2 / 3}$, where the capillary pressure is of order of $\sigma /\left(L \varepsilon^{2 / 3}\right)$. Then the ratio $\left(\sigma /\left(L \varepsilon^{2 / 3}\right)\right) /\left(\rho a L \varepsilon^{1 / 3}\right)=\sigma /\left(\rho a L^{2} \varepsilon\right)$ is much smaller than one when $T \gg 1.9 \times 10^{-3} \mathrm{~s}$. We conclude that viscous and surface tension effects can be neglected in the analysis of the plate-lifting problem after a short early stage, when the plate velocity is small, both in the main flow region and in the immediate vicinities of the plate edges. Note that the typical acceleration $a=20 \mathrm{~m} \mathrm{~s}^{-2}$, which comes from the experimental results in [3], was used in the above estimates. This value was chosen to satisfy another limitation of the present study, namely, that gravity can be neglected compared with the acceleration of the plate. For higher accelerations of the plate, the duration of the early stage, when viscous and surface tension effects should be included in the analysis, is smaller than in the reference case shown above.

\section{Second-order solution in the main flow region}

Outside the immediate vicinities of the plate edges the asymptotic solution of the problem (2)-(6) is sought in the form:

$\varphi(x, y, t)=\varphi_{0}(x, y, t)+\varepsilon \varphi_{1}(x, y, t)+O\left(\varepsilon^{2}\right), \quad \zeta(x, t)=\zeta_{0}(x, t)+\varepsilon \zeta_{1}(x, t)+O\left(\varepsilon^{2}\right)$.

Substituting (7) in Eqs. (2)-(6) and collecting terms without $\varepsilon$, we arrive at the boundary-value problem with respect to $\varphi_{0}(x, y, t)$ and $\zeta_{0}(x, t)$. Alternatively, the corresponding problem can be obtained by setting $\varepsilon=0$ in the system (2)-(6). The resulting mixed boundary-value problem has the solution:

$\varphi_{0}(x, y, t)+\mathrm{i} \psi_{0}(x, y, t)=\mathrm{i} t\left(\sqrt{z^{2}-1}-z\right), \quad \frac{\partial \varphi_{0}}{\partial x}-\mathrm{i} \frac{\partial \varphi_{0}}{\partial y}=\mathrm{i} t\left(\frac{z}{\sqrt{z^{2}-1}}-1\right)$,

where $z=x+\mathrm{i} y, \psi_{0}(x, y, t)$ is the leading-order stream function, and the branch of $\sqrt{z^{2}-1}$ is selected in such a way that $\sqrt{z^{2}-1}$ is real and positive at $z=x+\mathrm{i} 0, x>1$. It is seen that the elevation of the free surface $\zeta_{0}(x, t)$ to leading 
order is given by the linearized kinematic boundary condition, $\partial \zeta_{0} / \partial t=\left(\partial \varphi_{0} / \partial y\right)(x, 0, t)=t\left(1-x / \sqrt{x^{2}-1}\right)$, where $x>1$. This equation gives $\zeta_{0}(x, t)=\frac{1}{2} t\left(\partial \varphi_{0} / \partial y\right)(x, 0, t)$ and $\partial^{2} \zeta_{0} / \partial t^{2}=t^{-1}\left(\partial \varphi_{0} / \partial y\right)(x, 0, t)$. To leading order, the vertical velocity of the free surface and the free-surface deflection are square-root singular at the edge of the plate, $x=1$. Close to the plate edge, $x \rightarrow 1, y \rightarrow 0$, the potential $\varphi_{0}(x, y, t)$ and the free-surface shape $\zeta_{0}(x, t)$ behave as

$\varphi_{0}(x, y, t) \sim t \sqrt{2 R} \sin \left(\frac{\theta}{2}\right), \quad \zeta_{0}(x, t) \sim-\frac{t^{2}}{\sqrt{8 R}}$,

where $x=1+R \cos \theta, y=-R \sin \theta, 0 \leq \theta \leq \pi$ and $R \rightarrow 0$. Here $\theta=0$ corresponds to the free surface and $\theta=\pi$ to the surface of the plate.

Collecting terms with $\varepsilon$ in Eqs. (2)-(6), we obtain the boundary-value problem with respect to the potential $\varphi_{1}(x, y, t)$ and the first-order correction to the free-surface shape, $\zeta_{1}(x, t)$. Note that in the asymptotic expansions (7), we do not account for variation of the wetted area in time, setting $c(t, \varepsilon)=1$. The boundary condition (3), $\varphi_{y}\left(x, \varepsilon t^{2} / 2, t\right)=t$, and the asymptotic expansions (7) provide

$\varphi_{y}(x, 0, t)+\frac{1}{2} \varepsilon t^{2} \varphi_{y y}(x, 0, t)+O\left(\varepsilon^{2}\right)=t$,

$\varphi_{0 y}+\varepsilon\left(\varphi_{1 y}+\frac{1}{2} t^{2} \varphi_{0 y y}\right)=t+O\left(\varepsilon^{2}\right)$,

and finally

$\varphi_{1 y}=\frac{t^{2}}{2} \varphi_{0 x x} \quad(y=0, \quad|x|<1)$.

The dynamic boundary condition (4) yields

$\varphi_{t}(x, \varepsilon \zeta(x, t), t)+\frac{1}{2} \varepsilon|\nabla \varphi|^{2}=0$,

$\varphi_{t}(x, 0, t)+\varphi_{t y}(x, 0, t) \varepsilon \zeta+\frac{1}{2} \varepsilon|\nabla \varphi|^{2}=O\left(\varepsilon^{2}\right)$,

$\varphi_{0 t}+\varepsilon\left(\varphi_{1 t}+\zeta_{0} \varphi_{0 t y}+\frac{1}{2}\left|\nabla \varphi_{0}\right|^{2}\right)=O\left(\varepsilon^{2}\right)$

and thus

$\varphi_{1 t}=-\zeta_{0} \varphi_{0 y t}-\frac{1}{2} \varphi_{0 y}^{2} \quad(y=0, \quad|x|>1)$,

where

$\zeta_{0} \varphi_{0 y t}=\frac{t}{2} \varphi_{0 y} \zeta_{0 t t}=\frac{1}{2} \varphi_{0 y}^{2}$.

Notice that (8) has been used to eliminate $\varphi_{0 x}$ at the free surface. Finally,

$\varphi_{1 t}=-\varphi_{0 y}^{2} \quad(y=0, \quad|x|>1)$.

Correspondingly, the kinematic condition (4) to order $O(\varepsilon)$ gives

$\zeta_{1 t}=\varphi_{1 y}(x, 0, t) \quad(|x|>1)$.

It is convenient to decompose the first-order potential $\varphi_{1}(x, y, t)$ as

$\varphi_{1}(x, y, t)=\frac{t}{3}\left(\varphi_{0 x}^{2}-\varphi_{0 y}^{2}\right)+\frac{t^{3}}{6} \phi_{1}(x, y)$,

where $\phi_{1}(x, y)$ satisfies the Laplace equation for $y<0$, is equal to zero at the surface $y=0,|x|>1$, and obeys the boundary condition:

$\phi_{1 y}=\left(1-x^{2}\right)^{-3 / 2} \quad(y=0, \quad|x|<1)$, 
on the plate surface. Note that all terms in (13) are singular at the plate edges, $x= \pm 1, y=0$. The function $\phi_{1}(x, y)$ is not uniquely defined. In particular, on the plate, $|x|<1, y=0$,

$$
\begin{aligned}
& \phi_{1}(x, 0)=\frac{2 / \pi-C_{1}}{\sqrt{1-x^{2}}}+\frac{x}{\pi \sqrt{1-x^{2}}} \ln \left(\frac{1-x}{1+x}\right), \\
& \varphi_{1}(x, 0, t)=\frac{t^{3}}{6}\left(2 \frac{2 x^{2}-1}{1-x^{2}}+\phi_{1}(x, 0)\right),
\end{aligned}
$$

and the first-order correction to the shape of the free surface is

$\zeta_{1}(x, t)=\frac{t^{4}}{24\left(x^{2}-1\right)^{3 / 2}}\left(C_{1} x-\frac{1}{\pi} \ln \left(\frac{x-1}{x+1}\right)\right)$,

where $C_{1}$ is an undetermined real constant. The boundary condition (11) yields

$\varphi_{1}(x, 0, t)=-\frac{t^{3}}{3\left(x^{2}-1\right)}\left(\sqrt{x^{2}-1}-x\right)^{2} \quad(x>1)$.

Equations (15) and (17) show that

$\varphi_{1}(x, 0, t) \sim \frac{t^{3}}{3} \frac{1}{1-x^{2}} \quad(x \rightarrow 1)$.

The pressure distribution along the moving plate is given by

$p(x, 0, t)=-\sqrt{1-x^{2}}-\frac{\varepsilon t^{2} / 2}{1-x^{2}}\left(3 x^{2}+\frac{1}{\pi} x \sqrt{1-x^{2}} \ln \left(\frac{1-x}{1+x}\right)+\left(\frac{2}{\pi}-C_{1}\right) \sqrt{1-x^{2}}\right)+o\left(\varepsilon^{2}\right)$.

The second-order pressure distribution (19) is not integrable at the plate edges, $x \pm 1$. Also this pressure depends on a still undetermined constant $C_{1}$ which defines the asymptotic behaviour of $\zeta_{1}(x, t)$ at infinity (see Eq. (16)). This constant is expected to be obtained through matching the outer solution (7) with the inner solution describing the details of the flow near the plate edges. However, the constant $C_{1}$ does not appear in the asymptotic formula (18). This implies that the matching condition containing $C_{1}$ should involve at least the second-order inner solution. Therefore, the second-order pressure (19) and the corresponding second-order hydrodynamic force cannot be determined uniquely by means of only the second-order outer solution and the leading-order inner solution. It will be shown below that even the leading-order flow close to the plate edges as $\varepsilon \rightarrow 0$ is very complicated and can be studied only by numerical means at present.

Note that the term with $\ln ((1-x) /(1+x))$ in the first-order velocity potential (15) does not appear in the counterpart problem of the floating plate impact with non-zero initial speed (see [18]), but it does appear in the second-order Wagner problem of water entry [19]. Note also that the term with $1 / \sqrt{1-x^{2}}$ in (15) corresponds to an eigen solution of the exit problem in the leading order. That is, the asymptotic expansions (7) should be considered as formal and, in principle, terms of the kind $\varepsilon^{q} \varphi_{q}(x, y, t)$ could be included in (7) with $0<q<1$. The potentials $\varphi_{q}$ satisfy the boundary-value problem with zero boundary conditions: $\varphi_{q}=0$, where $y=0,|x|>1$, and $\varphi_{q y}=0$, where $y=0,|x|<1$. The non-zero solutions of this homogeneous problem are well known (see [18]). They are singular at $x= \pm 1, y=0$, the least singular potential being

$\varphi_{q}+\mathrm{i} \psi_{q}=\frac{\mathrm{i} C_{\mathrm{e}}(t)}{\sqrt{z^{2}-1}}$,

where $C_{\mathrm{e}}(t)$ is an undetermined real function of time. The corresponding contributions of the eigen potential (20) to the potential on the plate surface,

$\varepsilon^{q} \varphi_{q}(x, 0, t)=-\frac{\varepsilon^{q} C_{\mathrm{e}}(t)}{\sqrt{1-x^{2}}} \quad(|x|<1)$,

to the shape of the free surface,

$\varepsilon^{q} \zeta_{q}(x, t)=\frac{\varepsilon^{q} x}{\left(x^{2}-1\right)^{3 / 2}} \int_{0}^{t} C_{\mathrm{e}}(\tau) \mathrm{d} \tau \quad(x>1)$, 
and to the pressure distribution along the plate,

$\varepsilon^{q} \varphi_{q t}(x, 0, t)=-\varepsilon^{q} \frac{C_{e}^{\prime}(t)}{\sqrt{1-x^{2}}} \quad(|x|<1)$,

appear in the leading order. The power $q$ and the function $C_{e}(t)$ in (20)-(23) are undetermined and will be obtained using the matching condition between the second-order outer solution (7) with corrections (21)-(23) and the leadingorder inner solution, which will be studied in the next section. The small-time solution derived in this section is not valid near the plate edges, where an inner solution is needed to correct the flow and the pressure distribution.

\section{The inner flow close to the plate edges}

The leading-order inner solution of the problem (1)-(6) is derived by using the stretched inner variables $\xi$ and $\eta$

$x=1+\varepsilon^{k} \xi, \quad y=\varepsilon^{k} \eta, \quad r=\sqrt{\xi^{2}+\eta^{2}}$,

where $k>0$ and $\varepsilon \rightarrow 0$. The leading-order solution near the plate edges (9) predicts the following scaling of the velocity potential and the free-surface deflection:

$\varphi=\varepsilon^{k / 2} \phi(\xi, \eta, t, \varepsilon), \quad \zeta=\varepsilon^{-k / 2} H(\xi, t, \varepsilon)$.

The exponent $k$ is selected in such a way that the quadratic term in the Bernoulli equation (1),

$\frac{1}{2} \varepsilon|\nabla \varphi|^{2}=\frac{1}{2} \varepsilon^{1-k}|\nabla \phi|^{2}$,

and the linear term, $\varphi_{t}=\varepsilon^{k / 2} \phi_{t}$, are of the same order as $\varepsilon \rightarrow 0$. This gives $1-k=k / 2$ with the solution $k=2 / 3$.

In a small vicinity of the plate edge, $x=1$, of order $R=O\left(\varepsilon^{2 / 3}\right)$, the pressure (1) reads

$p(\xi, \eta, t)=-\varepsilon^{1 / 3}\left(\phi_{t}+\frac{1}{2}|\nabla \phi|^{2}\right)$.

The body boundary condition (3) provides

$\varepsilon^{-1 / 3} \phi_{\eta}=t \quad\left(\eta=\frac{1}{2} \varepsilon^{1 / 3} t^{2}, \quad \xi<c_{0}(t)\right)$,

where $\xi=c_{0}(t), c_{0} \leq 0$, is the position of the contact point. This boundary condition yields that, with accuracy $O\left(\varepsilon^{1 / 3}\right)$ as $\varepsilon \rightarrow 0$, the boundary condition can be simplified and imposed at $\eta=0$ :

$\phi_{\eta}=0 \quad\left(\eta=0, \xi<c_{0}(t)\right)$.

The kinematic and dynamic boundary conditions keep their forms in the inner variables (see also (26)):

$\phi_{t}+\frac{1}{2}|\nabla \phi|^{2}=0, \quad H_{t}+H_{\xi} \phi_{\xi}=\phi_{\eta} \quad(\eta=H(\xi, t))$.

Notice that the function $H(\xi, t)$ in (25) can be a multi-valued function of $\xi$. The leading-order inner solution (25) should be matched with the leading-order outer solution (9),

$\phi \sim t \sqrt{2 r} \sin \left(\frac{\theta}{2}\right), \quad H(\xi, t) \sim-\frac{t^{2}}{\sqrt{8 \xi}} \quad(r \rightarrow \infty)$.

Therefore, in the leading order as $\varepsilon \rightarrow 0$, the flow near the plate edges is linear and with an unknown position of the surface. This problem can be solved only by numerical means. In this section, we derive some theoretical results for the solution of the nonlinear inner problem. Note that the inner problem is as complicated as the original problem (2)-(6). The only simplification concerns the body boundary condition (27), but this simplification is not significant. On the other hand, the inner solution is driven by the trivial far-field conditions (29) which are much more complicated than the original conditions (5). 
Fig. 1 a Numerical domain. In the simulations reported here, $R_{\infty}=5000$. b Sketch of the free surface and the coordinate systems (a)
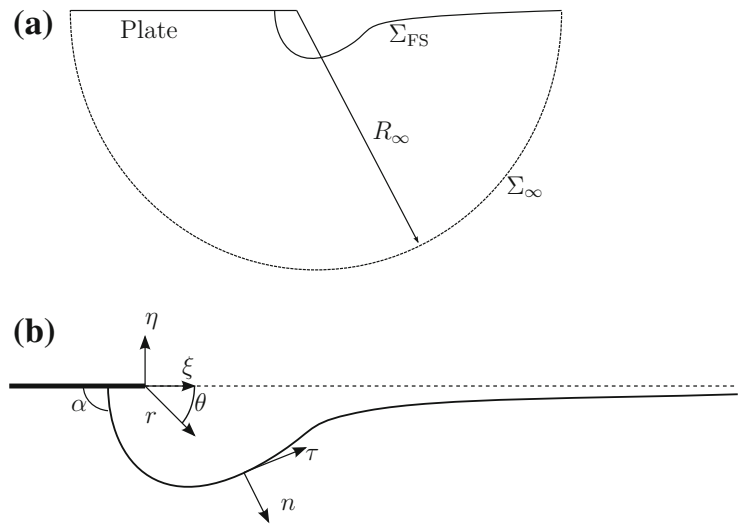

The solution of the formulated inner problem is self-similar with the variables

$\xi=t^{4 / 3} \lambda, \quad \eta=t^{4 / 3} \mu, \quad \phi=t^{5 / 3} \Phi(\lambda, \mu), \quad H=t^{4 / 3} h(\lambda)$.

In the self-similar variables (30), the boundary-value problem of the inner flow reads

$\nabla^{2} \Phi=0 \quad$ (in the flow region),

$\Phi_{\mu}=0 \quad\left(\mu=0, \lambda<\lambda_{c}\right)$,

$\frac{5}{3} \Phi-\frac{4}{3}\left(\lambda \Phi_{\lambda}+\mu \Phi_{\mu}\right)+\frac{1}{2}|\nabla \Phi|^{2}=0$, and

$\frac{4}{3}\left(h-\lambda h_{\lambda}\right)+h_{\lambda} \Phi_{\lambda}=\Phi_{\mu} \quad$ (on the free surface, $\left.\quad \mu=h(\lambda)\right)$,

$\Phi \sim \sqrt{2 \rho} \sin \left(\frac{\theta}{2}\right) \quad(\rho \rightarrow \infty), \quad h \sim-(8 \lambda)^{-1 / 2} \quad(\lambda \rightarrow \infty)$

where $\rho^{2}=\lambda^{2}+\mu^{2}$ and $\lambda_{c} \leq 0, c_{0}(t)=\lambda_{c} t^{4 / 3}$. The expected shape of the surface is shown in Fig. 1. The selfsimilar and linear flow close to the plate edge is described by a system of equations plus boundary conditions which are similar to those derived by Iafrati and Korobkin [17] in the inner problem of floating plate impact. However, in their case, the coefficients at the free-surface boundary conditions and in the conditions at infinity are different. The hydrodynamic pressure along the plate, $p=-\varepsilon^{1 / 3} t^{2 / 3} P(\lambda, 0)$, is given by

$P(\lambda, 0)=\frac{5}{3} \Phi-\frac{4}{3} \lambda \Phi_{\lambda}+\frac{1}{2} \Phi_{\lambda}^{2}$,

where the potential and its derivatives are evaluated at $(\lambda, 0)$. Similar to the analysis in [17], it is possible to integrate the dynamic boundary condition on the free surface by using a modified velocity potential

$S(\lambda, \mu)=\frac{2}{3} \rho^{2}-\Phi(\lambda, \mu)$.

The new unknown function (33) satisfies the equation

$\nabla^{2} S=\frac{8}{3}$

in the flow region, its normal derivative $\partial S / \partial n$ is equal to zero on both the plate, $\mu=0, \lambda<\lambda_{c}$, and on the free surface, $\mu=h(\lambda)$, and the dynamic boundary condition provides 
$S_{\tau}^{2}=\frac{10}{3} S-\frac{4}{9} \rho^{2}$,

where $S_{\tau}$ is the tangential derivative along the free surface. Equation (35) allows us to calculate the distribution of the velocity potential along the free surface, if the shape of the free surface is known. Equation (32) in terms of the function $S(\lambda, \mu)$ yields the pressure along the plate

$P(\lambda, 0)=\frac{1}{2} S_{\lambda}^{2}(\lambda, 0)-\frac{5}{3} S(\lambda, 0)+\frac{2}{9} \lambda^{2} \quad\left(\lambda<\lambda_{c}\right)$.

Equation (35) in terms of the velocity potential on the free surface, $\Phi_{f}(\lambda)=\Phi(\lambda, h(\lambda))$, provides

$\Phi_{f \lambda}^{2}+\frac{10}{3} \Phi_{f}(\lambda)\left(1+h_{\lambda}^{2}\right)-\frac{8}{3}\left(\lambda+h h_{\lambda}\right) \Phi_{f \lambda}=\frac{16}{9}\left(\lambda h_{\lambda}-h\right)^{2}$.

The condition at infinity, $h \sim-(8 \lambda)^{-1 / 2}$ as $\lambda \rightarrow+\infty$, shows that the right-hand side of (37) behaves at infinity as $1 /(2 \lambda)$, which gives $\Phi_{f}(\lambda) \sim 1 /(12 \lambda)$ as $\lambda \rightarrow \infty$. It can be shown that the second-order outer solution (7) provides the same results in the leading order as $\varepsilon \rightarrow 0$ and $x \rightarrow 1^{+}$. By using these results we can obtain the far-field asymptotic behaviour of the potential $\Phi(\lambda, \mu)$ :

$\Phi(\lambda, \mu)=\sqrt{2 \rho} \sin \left(\frac{\theta}{2}\right)+\frac{C_{*}}{\sqrt{\rho}} \sin \left(\frac{\theta}{2}\right)-\frac{\cos \theta}{6 \rho}+O\left(\rho^{-3 / 2}\right)$.

The term with the undetermined constant $C_{*}$ in (38) is the eigen solution of the inner problem in the far field. On the plate, $\theta=\pi$, Eq. (38) provides

$\Phi(\lambda, 0)=\sqrt{-2 \lambda}+\frac{C_{*}}{\sqrt{-\lambda}}-\frac{1}{6 \lambda}+O\left(|\lambda|^{-3 / 2}\right)$,

and the far-field behaviour of the pressure (32) is

$P(\lambda, 0)=\sqrt{-2 \lambda}+\frac{3 C_{*}}{\sqrt{-\lambda}}-\frac{3}{4 \lambda}+O\left(|\lambda|^{-3 / 2}\right)$ as $\lambda \rightarrow \infty$.

In the present analysis, we assume that the pressure $P(\lambda, 0)$ is zero at the contact point $\lambda=\lambda_{\mathrm{c}}$ and the free surface is not tangential to the plate at the contact point. The normal derivative $S_{n}$ is equal to zero on the plate and on the free surface including the contact point, but the normal vectors at the contact point to the plate and to the free surface are different if the free surface is not tangential to the plate at this point. This gives $S_{\lambda}=0$ and $S_{\mu}=0$ at $\mu=0, \lambda=\lambda_{\mathrm{c}}$ for a non-singular solution. Then the condition (35) and the formula for the pressure (36) yield the inner potential at the contact point

$\Phi\left(\lambda_{\mathrm{c}}, 0\right)=\frac{8}{15} \lambda_{\mathrm{c}}^{2}, \quad \Phi_{\lambda}\left(\lambda_{\mathrm{c}}, 0\right)=\frac{4}{3} \lambda_{\mathrm{c}}$.

The coordinate $c(t, \varepsilon)$ of the contact point (see (1)-(6)) is related to the constant $\lambda_{\mathrm{c}}$ through (24) and (30):

$c(t, \varepsilon) \sim 1+\varepsilon^{2 / 3} t^{4 / 3} \lambda_{\mathrm{c}}$,

to leading order as $\varepsilon \rightarrow 0$. By using (41), it can be shown that the speed of the contact point is equal to the local speed of the flow at this point, to leading order. Note that the speed of the contact point was set to be equal to the double speed of the local flow at this point in [2]. This factor was found by comparing the theoretical predictions of the hydrodynamic forces with the numerical predictions from [3]. This factor has not been rigorously justified. In terms of the non-dimensional variables in (1)-(6), the corresponding asymptotic formula reads

$\varphi_{x}\left(c(t, \varepsilon), \frac{1}{2} \varepsilon t^{2}, t\right) \sim \frac{1}{\varepsilon} \frac{\mathrm{d} c}{\mathrm{~d} t} \quad(\varepsilon \rightarrow 0)$. 
Note that (38)-(41) can be helpful in numerical simulations of the inner problem and evaluation of the coefficient $C_{*}$ in (38). By means of the outer pressure distribution (19) with the correction (23) and the inner pressure (36), one can evaluate the force acting on the lifting plate by asymptotic integration of the pressure, as has been done in [18].

The exponent $q$ and the function $C_{\mathrm{e}}(t)$ in (23) are obtained by matching the eigen value term (21) along the plate to the second term with $C_{*}$ in (39). The result is $q=2 / 3$ and $C_{\mathrm{e}}(t)=-\sqrt{2} C_{*} t^{7 / 3}$. Then the correction (23) to the pressure distribution (19) reads

$$
\frac{7 \sqrt{2}}{3} C_{*} \frac{\left(\varepsilon t^{2}\right)^{2 / 3}}{\sqrt{1-x^{2}}} .
$$

Therefore, the pressure distribution on the main part of the plate is determined with accuracy $o(\varepsilon)$ up to two undetermined constants $C$ and $C_{*}$. These constants are obtained by matching the outer solution with the inner solution near the plate edges. In particular, the pressure at the centre of the plate is given by

$p\left(0, \frac{1}{2} \varepsilon t^{2}, t\right)=-1+\frac{7 \sqrt{2}}{3} C_{*}\left(\varepsilon t^{2}\right)^{2 / 3}+\frac{1}{2}\left(C-\frac{2}{\pi}\right) \varepsilon t^{2}+o(\varepsilon)$,

in the non-dimensional variables.

\section{Numerical analysis of the inner flow}

The potential nonlinear problem (27)-(29), where the position of the free surface is unknown $a$ priori, is solved in a numerical domain bounded by the free surface, $\Sigma_{\mathrm{FS}}(t)$, a semi-circular far-field boundary, $\Sigma_{\infty}$, with radius $R_{\infty}$, where the potential satisfies equation (29), and a flat plate lying on $\eta=0$, where the no-flux condition (27) is imposed. A sketch of the domain and coordinate system is shown in Fig. 1.

The self-similar solution (30) is sought by a time-marching method. In this method, the potential on the free surface, as well as the location of this surface, evolves in time according to the equations

$$
\begin{aligned}
& \frac{\mathrm{d} \phi}{\mathrm{d} t}=-\frac{1}{2}\left(\phi_{\tau}^{2}+\phi_{n}^{2}\right), \\
& \frac{\mathrm{d} \overrightarrow{\mathbf{x}}_{\mathrm{FS}}}{\mathrm{d} t}=\phi_{\tau} \vec{\tau}+\phi_{n} \overrightarrow{\mathbf{n}} .
\end{aligned}
$$

Note that these equations are in fact the boundary conditions (28) written in Lagrangian formulation, which is more convenient for numerical purposes.

The numerical algorithm is as follows: (i) for a given initial distribution of the potential $\phi$ along the free surface, Laplace's equation is solved to compute the normal velocity $\phi_{n}$ along the free surface. A first-order boundaryelement method has been used to this end, implemented with the library BEMLIB (see [20]); (ii) the tangential velocity on the boundary, $\phi_{\tau}$, is computed by differentiating the potential with respect to the arc-length parameter, $\tau$; (iii) finally, using a second-order Runge-Kutta method, the potential and the free surface are evolved according to (44) and (45). A pseudo-time step, $\Delta t$, was adjusted to ensure either a Courant-Friedrichs-Lewy condition of 0.25 or a maximum value $\Delta t_{\max }=10^{-3}$. Finally, after each time step, the free surface was re-meshed to guarantee a minimum panel size, $\Delta \tau=0.01$, at the contact point between the free surface and the plate. The panel size grows by a factor 1.0025 as one moves away from this point.

The evolution of the free surface computed with this algorithm is shown in Fig. 2 using the self-similar variables (30) for several values of the pseudo-time between $t \approx 5$ and $t \approx 43$, when the simulation was stopped (at that time, numerical instabilities in the far-field region of the free surface $\left(r \rightarrow R_{\infty}\right)$, close to $\Sigma_{\infty}$, started to develop). It can be observed that the free surface has nearly converged to a steady solution, except close to the corner that forms at around $\lambda \approx 0.1, \mu \approx-0.4$. Far from this corner, the free surface tends to the asymptotic value given by (31), $\mu \sim-1 / \sqrt{8 \lambda}$ (black line of the lower panel). To understand why the solution converges so slowly around the corner, it might be illustrative to plot the evolution of the normal derivative of the modified potential (33) analogous 

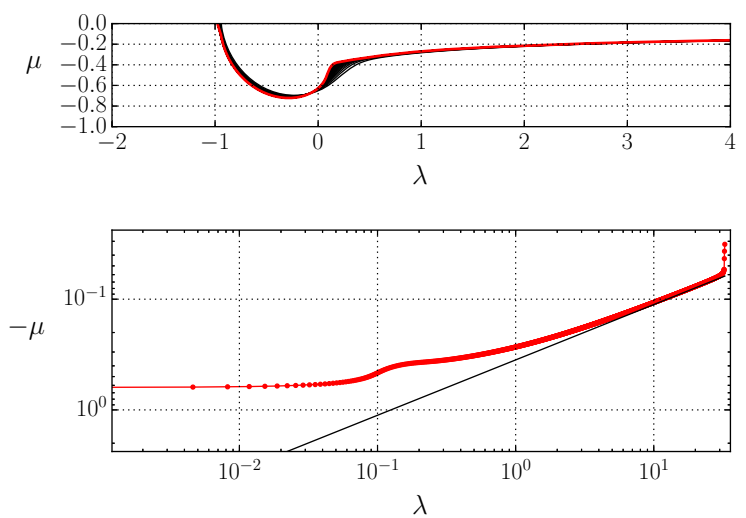

Fig. 2 Top panel evolution of the free surface during the timemarching procedure (black lines). The grey line (red in the online version) corresponds to the last time step. Notice how the surface has nearly converged, except at the corner near $(\lambda \approx 0.1$, $\mu \approx-0.4)$. Bottom panel converged free surface in the far field. The black thin line represents the asymptotic expression $h(\lambda) \sim-1 / \sqrt{8 \lambda}$. (Color figure online)

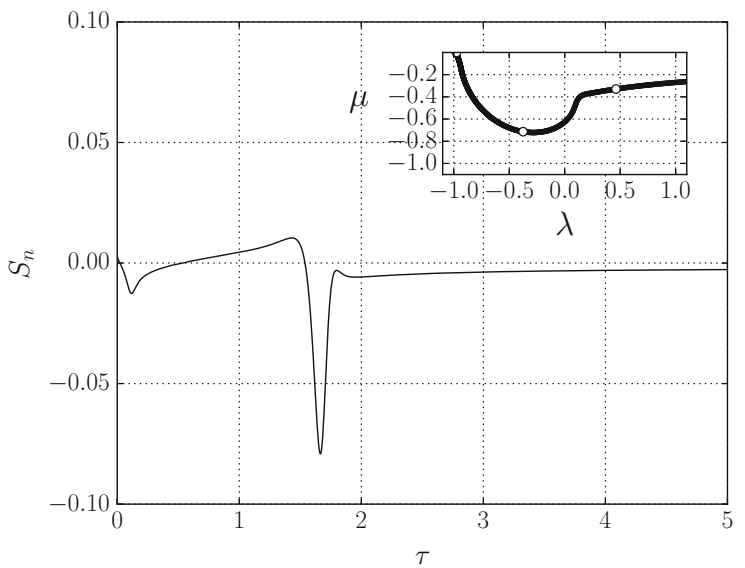

Fig. 3 Normal derivative of the modified potential, $S_{n}$. This magnitude should be zero when the self-similar solution is achieved. The inset shows a detail of the region close to the contact point, where the white circles mark the points $\tau=0,1$ and 2
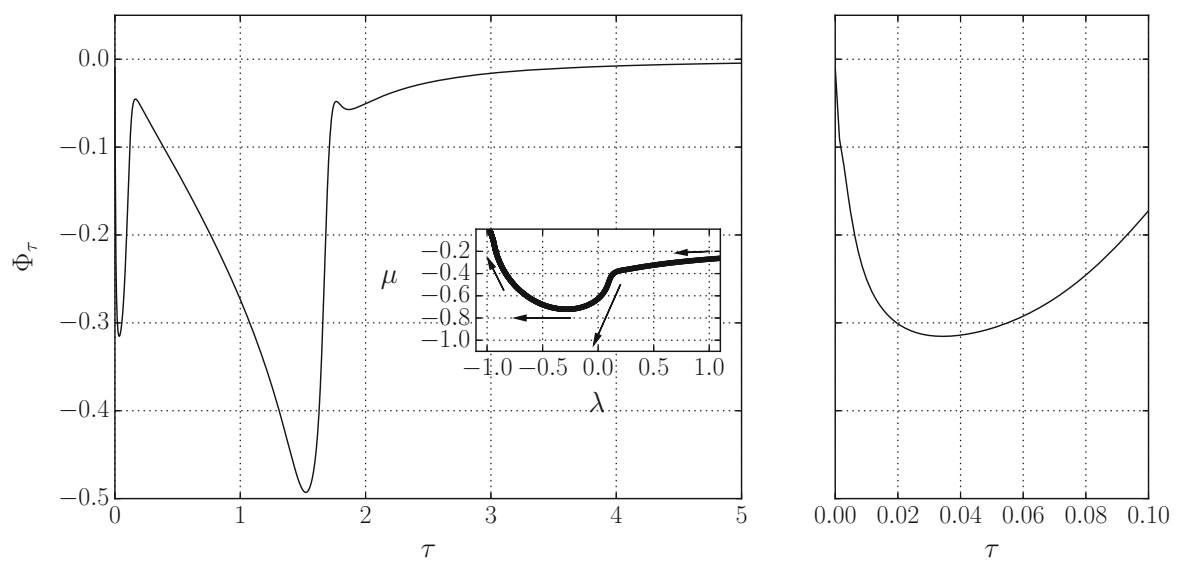

Fig. 4 Tangential velocity along the free surface corresponding to the last time step. The inset shows qualitatively the direction and modulus of the velocity. The right panel shows close up of the region close to the contact

to the one defined by Iafrati and Korobkin in [17] for the plate water-entry problem. Expressed in terms of this modified potential, the kinematic boundary condition from (31) turns into $S_{n}=0$ on the free surface. Thus, if the self-similar shape is reached, this condition should be fulfilled along the whole free surface. Figure 3 shows the distribution of $S_{n}$ along the free surface for the last time step. The normal derivative of the modified potential has converged to a value smaller than about $10^{-2}$, except for the spike at $\tau \approx 1.75$, corresponding to the corner (see inset).

The slow convergence near the corner is compatible with the existence of a discontinuity in the slope of the free surface at that point. In fact, Needham and co-workers showed that in an analogous system such singularities do exist [21]. In their particular problem, the flow sets in motion by an inclined plate that accelerates at a constant rate from rest, a corner is formed depending on the inclination angle of the plate, which precluded the numerical computation of the solution from that point on. In our case, the time-marching procedure allows us to infer the 


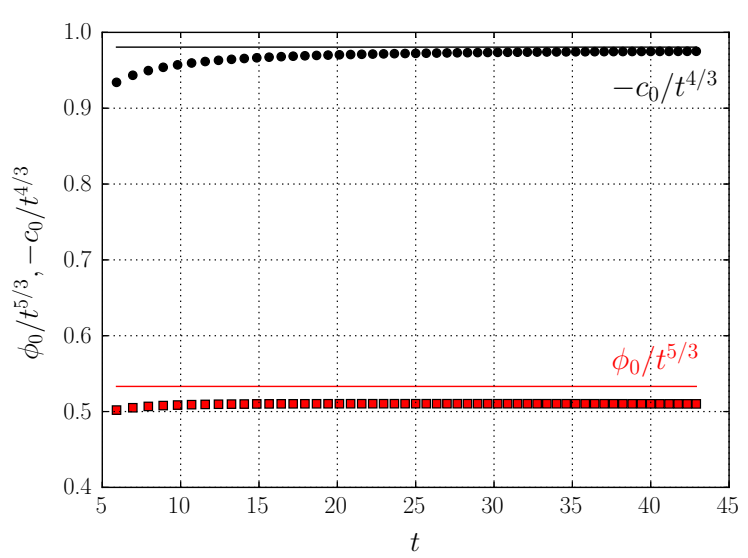

Fig. 5 Location of the contact point, $c_{0}$ (black circles), and the potential there, $\phi_{0}=\phi\left(c_{0}, 0\right)$ (red squares), rescaled with the time according to the self-similar scaling proposed above. The solid lines depict the theoretical value given by Eq. (41). Although both magnitudes follow this self-similar scaling, the potential exhibits some discrepancies with the theoretical value. (Color figure online)
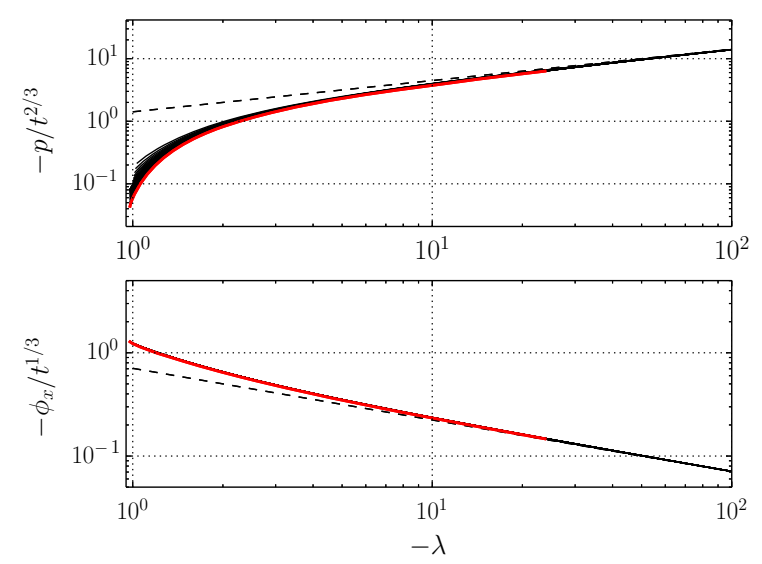

Fig. 6 Top panel evolution of the pressure distribution along the plate, plotted in self-similar variables, with the grey line (red in the on-line version) showing the last time step. The pressure converges to the asymptotic value (black dashed line), given by the leading-order term in Eq. (40). Bottom panel evolution of the velocity along the plate. The convergence is so fast that all the time steps shown (corresponding to the points in Fig. 5) collapse onto a single curve that, in the far-field limit, $\lambda \rightarrow \infty$, approaches the asymptote given by the leading order of Eq. (39). (Color figure online)

shape of the free surface with a reasonable accuracy, except obviously at the singular point and, as will be shown below, at the contact point.

Regarding the conditions for the formation of the singularity, although it would be tempting to think that, similar to what Needham and co-authors found in [21], there could exist a critical value of the contact angle between the plate and the free surface, $\alpha_{\mathrm{c}}$, that would yield a solution that is regular everywhere. However, this is not the case here. Indeed, Fig. 7 shows the final free surface, normal velocity of the modified potential, $S_{n}$, and dimensionless tangential velocity, $\Phi_{\tau}$, in the vicinities of the plate. It can be seen how, except very close to it, the structure of the flow is not affected by the choice of this contact angle. In particular, the corner found near $\tau=1.75$ is found for all the values of $\alpha$ explored. This points out that using plates with different wettability properties (hydrophobic or hydrophilic surface, for instance) would not affect significantly the structure of the flow described in this work, except, naturally, very close to the surface.

Presumably, the effect of the singular corner has also an effect on the contact point. Figure 4 displays the tangential velocity, $\Phi_{\tau}$, along the free surface. It can be seen how this velocity shows a peak close to the contact point, $\xi=c_{0}(t), \eta=0$, before becoming zero at the plate. Consequently, the convergence of the solution near the plate is also very slow. Figure 5 shows the evolution of the rescaled $x$ coordinate of the contact point, $c_{0}(t)$, and the potential at that point, $\phi_{0}(t)=\phi\left(c_{0}(t), 0, t\right)$ rescaled according to the proposed self-similar structure of the problem, i.e. $c_{0}(t) / t^{4 / 3}$ and $\phi_{0}(t) / t^{5 / 3}$. Although both magnitudes follow the self-similar scaling for $t>20$, the potential does not coincide with the theoretical value, exhibiting a relative error of about $5 \%$. In that figure, the grey solid line (red in the online version) is computed using the first equation (41), taking the value of $\lambda_{c}$ corresponding to the last time step. Conversely, the black solid line has been calculated with the second equation (41), using the value of $\Phi_{\lambda}\left(\lambda_{c}, 0\right)$ of the final integration time.

Finally, it is interesting to show the evolution of both the velocity, $\phi_{x}$, and pressure distribution, $p=-\phi_{t}-\frac{1}{2} \phi_{x}^{2}$, along the plate (see Fig. 6). Whereas the velocity converges very quickly to the self-similar distribution, the pressure near the contact takes longer to do so, specially close to the contact point. Most likely, this is an effect of the influence 

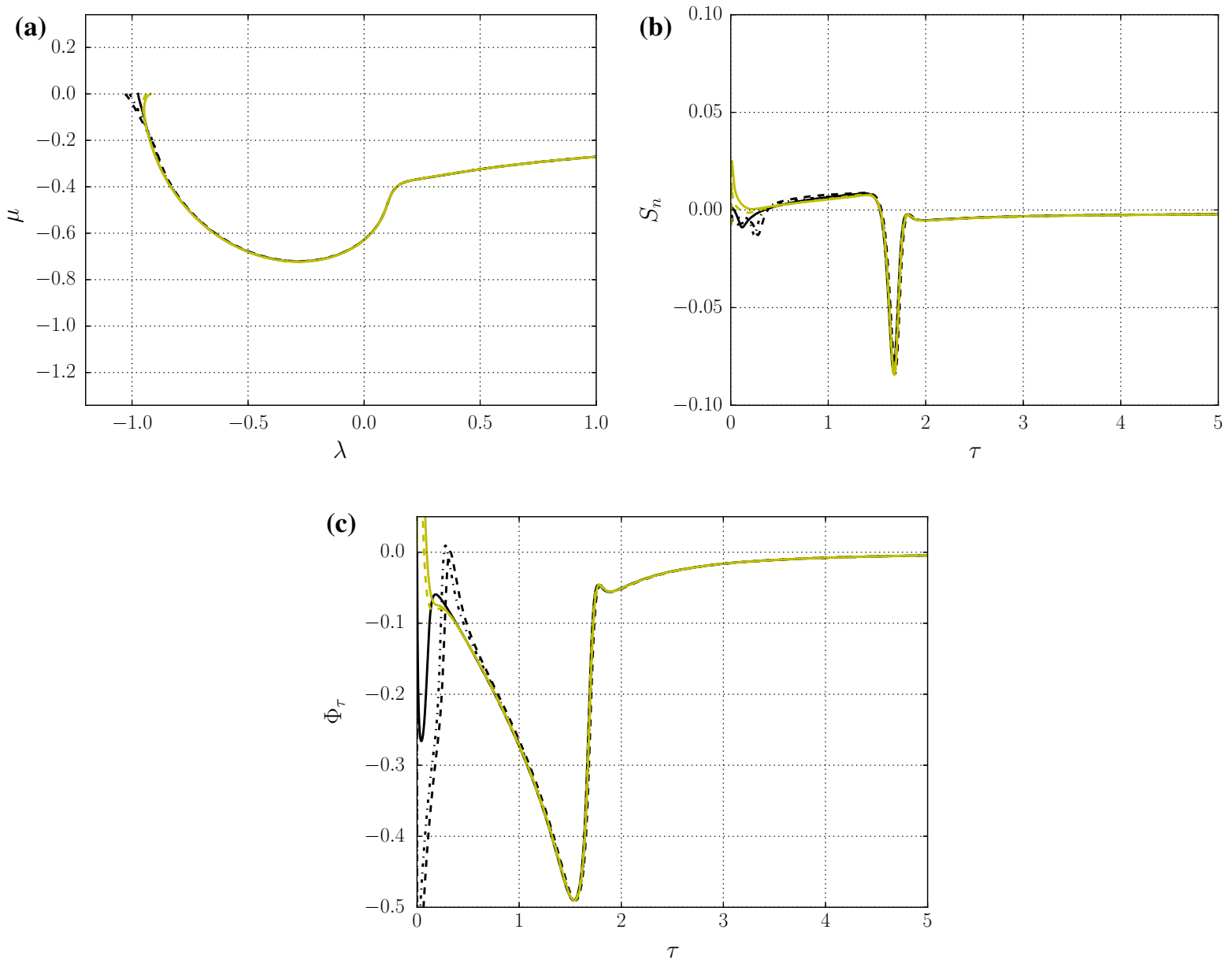

Fig. 7 a Free surface near the plate for different contact angles, namely $\alpha=45^{\circ}$ (black dashed line), $60^{\circ}$ (black dash-dotted line), $90^{\circ}$ (black solid line) $120^{\circ}$ (grey dashed line, yellow in the on-line version) and $152^{\circ}$ (solid line, yellow in the on-line version). Notice how, except close to the plate $(y=0)$, the different free surfaces nearly coincide. b Evolution of the parameter $S_{n}$ near the free surface. Notice the existence of the spike near $s \approx 1.7$ for all the contact angles explored. $\mathbf{c}$ Self-similar tangential velocity along the free surface. (Color figure online)

of the corner on this region discussed in the previous paragraph. Despite this, the pressure converges to the far-field solution, $p / t^{2 / 3} \sim-\sqrt{2 \lambda}$ (black dashed line).

\section{Conclusions}

The numerical solution of the problem exhibits the self-similar scaling proposed by the asymptotic analysis, which proves the validity of the theory to describe the flow close to the contact point between the plate and the free surface. Interestingly, some discrepancies arise, although they might be associated to the numerical error introduced by the existence of a singularity in the mathematical solution, very much like the one found by Needham et al. [21]. Although our numerical method, based on time-marching the flow in a pseudo-time, is at least able to describe the overall structure of the flow, it clearly fails to accurately accommodate the existence of the singularity. However, in contrast to the flow considered in reference [21], the corner found here seems to be insensitive to the particular choice of the contact angle, $\alpha$, as is illustrated in Fig. 7. 
Acknowledgments This work has been supported by the NICOP research grant "Fundamental Analysis of the Water Exit Problem" N62909-13-1-N274, through Dr. Woei-Min Lin. The authors wish to acknowledge the support of the Spanish Ministry of Economy and Competitiveness through grant DPI2014-59292-C3-1-P. The authors also acknowledge the support of the London Mathematical Society that funded a research stay of J. R-R at the University of East Anglia in the summer of 2014. The authors are grateful to the referees for their careful readings and comments, which helped to improve an earlier version of this paper.

Open Access This article is distributed under the terms of the Creative Commons Attribution 4.0 International License (http:// creativecommons.org/licenses/by/4.0/), which permits unrestricted use, distribution, and reproduction in any medium, provided you give appropriate credit to the original author(s) and the source, provide a link to the Creative Commons license, and indicate if changes were made.

\section{References}

1. Reis PM, Jung S, Aristoff JM, Stocker R (2010) How cats lap: water uptake by Felis catus. Science 330(6008):1231-1234

2. Korobkin AA (2013) A linearized model of water exit. J Fluid Mech 737:368-386

3. Piro DJ, Maki KJ (2011) Hydroelastic wedge entry and exit. In: Eleventh international conference on fast sea transport, Honolulu. 26-29 Sept 2011

4. Korobkin A, Khabakhpasheva TI, Maki KJ (2014) Water-exit problem with prescribed motion of a symmetric body. In: Proceedings of the 29th international workshop on water waves and floating bodies, Osaka, pp 117-120

5. Korobkin A, Maki KJ, Rodríguez-Rodríguez J, Khabakhpasheva TI (2014) Oscillations of an elastically supported body in partial contact with water. In: Proceedings of 17th U.S. national congress on theoretical and applied mechanics, Michigan State University. 15-20 June 2014

6. Greenhow M (1988) Water-entry and -exit of a horizontal circular cylinder. Appl Ocean Res 10(4):191-198

7. Greenhow M, Moyo S (1997) Water entry and exit of horizontal circular cylinders. Philos Trans R Soc A 355:551-563

8. Oliver JM (2002) Water entry and related problems. PhD thesis, University of Oxford

9. Baarholm RJ (2001) Theoretical and experimental studies of wave impact underneath decks of offshore platforms. PhD thesis, Department of Marine Hydrodynamics, NTNU, Trondheim

10. Scolan YM, Remy F, Thibault B (2006) Impact of three-dimensional standing waves on a flat horizontal plate. In: Proceedings 21st IWWWFB, Loughborough, pp 165-168

11. Faltinsen OM, Landrini M, Greco M (2004) Slamming in marine applications. J Eng Math 48:187-217

12. Piro DJ, Maki KJ (2013) Hydroelastic analysis of bodies that enter and exit water. J Fluids Struct 37:134-150

13. Iafrati A, Korobkin AA (2011) Asymptotic estimates of hydrodynamic loads in the early stage of water entry of a circular disk. J Eng Math 69(2-3):199-224

14. Tassin A, Piro DJ, Korobkin AA, Maki KJ, Cooker MJ (2013) Two-dimensional water entry and exit of a body whose shape varies in time. J Fluids Struct 40:317-336

15. Greenhow M, Lin W (1983) Non-linear free surface effects: experiments and theory. Report Number 83-19, Department of Ocean Engineering, Massachusetts Institute of Technology. (Photos from this report have been scanned from the negatives and are available at: http://www.researchgate.net/profile/Martin_Greenhow/publications)

16. Zhu X (2006) Application of the CIP method to strongly nonlinear wave-body interaction problems. PhD thesis, Norwegian University of Science and Technology

17. Iafrati A, Korobkin AA (2004) Initial stage of flat plate impact onto liquid free surface. Phys Fluids 16(7):2214-2227

18. Iafrati A, Korobkin AA (2008) Hydrodynamic loads during early stage of flat plate impact onto water surface. Phys Fluids 20(8):082104

19. Korobkin AA (2007) Second-order Wagner theory of wave impact. J Eng Math 58(1-4):121-139

20. Pozrikidis C (2002) A practical guide to boundary element methods with the software library BEMLIB. CRC Press, Boca Raton

21. Needham DJ, Chamberlain PG, Billingham J (2008) The initial development of a jet caused by fluid, body and free-surface interaction. Part 3. An inclined accelerated plate. Q J Mech Appl Math 61:581-614 